\title{
Madelung's disease in a patient with chronic renal insufficiency: a case report and review of literature
}

\author{
Beata Bergler-Czop¹, Dominika Wcisło-Dziadecka², Ligia Brzezińska-Wcisło ${ }^{1}$ \\ 'Department of Dermatology, Medical University of Silesia, Katowice, Poland \\ Head of Department: Prof. Ligia Brzezińska-Wcisło MD, PhD \\ ${ }^{2}$ Institute of Structural Research of Skin, Department of Cosmetology, Medical University of Silesia, Sosnowiec, Poland \\ Head of Department: Krzysztof Jasik PhD
}

Postep Derm Alergol 2014; XXXI, 2: 121-124

DOI: $10.5114 / p d i a .2014 .40922$

\begin{abstract}
Madelung's disease (benign symmetric lipomatosis, Launois-Bensaude syndrome) was described for the first time in the middle of the $19^{\text {th }}$ century. This disorder concerns mainly men between 30 and 60 years of age. Patients often suffer from coexisting ailments, such as hepatic function disorders, polyneuropathy, diabetes, gynecomastia, hyperuricemia and deviations of lipid management parameters. Treatment of benign symmetric lipomatosis consists mainly in fat tissue sucking or injection lipolysis. Patients often have to wear special clothes correcting the deformed figure. We are presenting a case of a female patient with diagnosed Madelung's disease, without alcohol abuse. Such abuse is reported in $90 \%$ of patients with this disease. Nevertheless, the patient has other typical (and described in the literature) hepatic and pancreatic function impairments, but also glomerular nephritis, which very rarely co-exists with benign symmetric lipomatosis.
\end{abstract}

Key words: Madelung's disease, lipomatosis, treatment.

\section{Introduction}

Madelung's disease (benign symmetric lipomatosis, Launois-Bensaude syndrome) was described for the first time in the middle of the $19^{\text {th }}$ century. This disorder concerns mainly men between 30 and 60 years of age. The direct cause of the disease is unknown. Impaired lipid metabolism and inhibition of lipidosis most probably result in adipose tissue proliferation and adipogenesis, mainly around the neck, shoulders and the abdominal area, which leads to considerable deformation of body contours. Due to the dominating fat tissue layout, three types of the disorder have been distinguished: type Iinvolving the neck (Fetthals), type II - involving shoulders (pseudo-athletic type), and type III - involving the abdomen (gynecoid type) [1].

Patients often suffer from coexisting ailments, such as hepatic function disorders, polyneuropathy, diabetes, gynecomastia, hyperuricemia and deviations of lipid management parameters.

Treatment of benign symmetric lipomatosis consists mainly in fat tissue sucking or injection lipolysis. Patients often have to wear special clothes correcting the deformed figure [2, 3].
We present a case of a 54-year-old woman with diagnosed Madelung's disease and coexisting glomerular nephritis.

\section{Case report}

The patient is 54 years old. The first skin lesions, characterized by symmetric adipose tissue proliferation on both arms, appeared 7 years earlier. The changes increased gradually. When the patient was 33 years old, she was diagnosed with common psoriasis. For the last 3 years, she has been a patient of the Nephrology Department due to chronic renal failure, which was diagnosed as a result of pyelonephritis. In 2004, the patient was hospitalised in the Internal and Metabolic Diseases Chair and Clinic, where the diagnosis of benign symmetric lipomatosis was suggested for the first time. The following were also observed: mixed hyperlipidemia, arterial hypertension, hypertrophic cardiomyopathy and chronic circulatory failure. Within 7 years of the disease, the patient gained $22 \mathrm{~kg}$. The medical history of the patient does not reveal alcohol abuse. In 2011, she was admitted to the Dermatology Department of the Medical Univer-

Address for correspondence: Beata Bergler-Czop MD, PhD, 2a Leśna St, 42-624 Ossy, Poland, phone/fax: +48 322840877 , e-mail: bettina2@tlen.pl Received: 8.02.2013, accepted: 23.06.2013. 


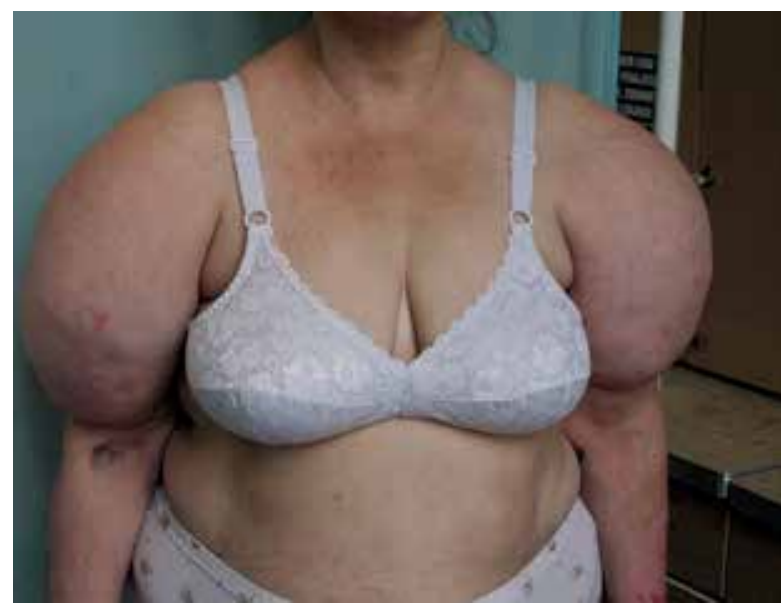

Figure 1. Patient 54 years old, massive, soft, indolent and symmetric tissue proliferation around both shoulders (reputed athletic figure)

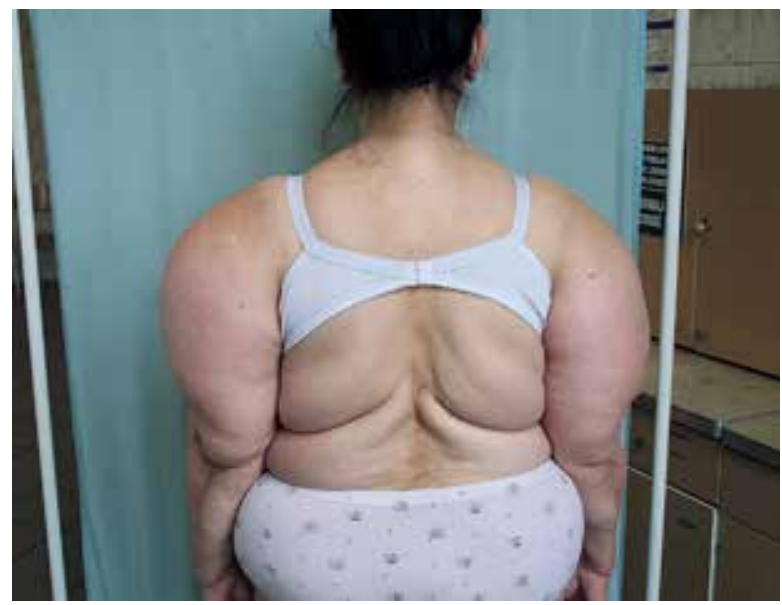

Figure 2. Patient 54 years old, symmetric tissue proliferation, back

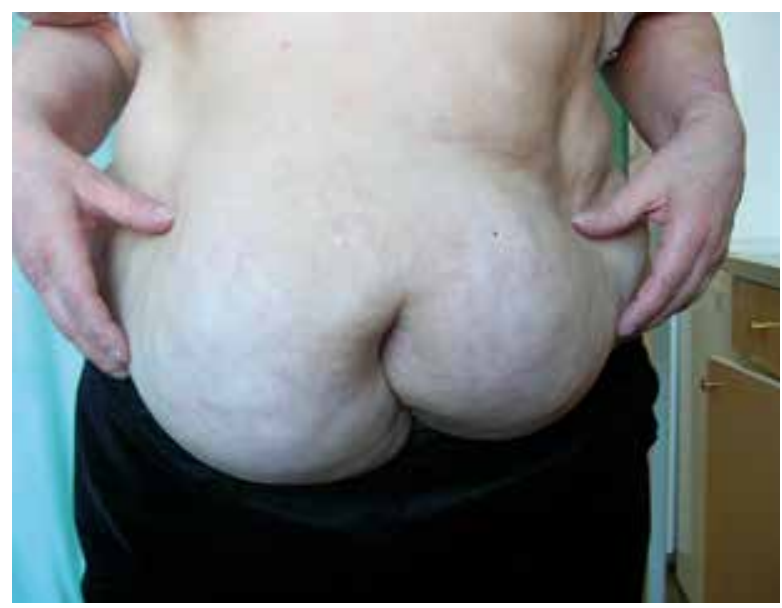

Figure 3. Patient 54 years old, abdomen sity of Silesia in Katowice in order to undergo complete diagnostic procedures related to skin lesions.

When admitted, the patient had massive, soft, indolent and symmetric tissue proliferation around both shoulders (pseudo-athletic figure). Physicians also observed adipose tissue proliferation in the abdominal area, around buttocks and thighs. Above elbow joints, on forearms and around the hypogastrium, there were single, confluent lumps of erythematous-exfoliating character. Peripheral lymph nodes were not enlarged. Mucous membranes, nails and the scalp were free of changes (Figures 1-3).

Laboratory tests revealed: erythrocyte sedimentation rate (ESR) 29, $\gamma$-glutamyl transpeptidase (GGTP) 118 (normal range up to $24 \mathrm{IU} / \mathrm{I}$ ), amylase 141 (normal range up to $100 \mathrm{IU} / \mathrm{I}$ ), and creatinine 118 (normal range up to 96 $\mu \mathrm{mol} / \mathrm{l})$. General urine analysis revealed erythrocyturia and proteinuria. Blood morphology with peripheral blood smear, iron level, electrolytes, aspartate aminotransferase (AST), alanine aminotransferase (ALT), bilirubin, urea, protein, protein electrophoresis, creatine phosphokinase (CPK), aldolase, triglycerides, cholesterol, joint reactions (Latex-R, Waaler-Rose Reaction, ASO) and glucose level were normal. CEA, CA 125 tumour markers fell within the normal range.

A chest X-ray: increased vascular pattern, enlarged left ventricle of the heart, slightly vascular hili. Abdominal ultrasound: an additional spleen, cortical layer of both kidneys with increased echogenicity, kidneys of nonsymetric shape. Ultrasound of soft tissues in shoulders: echogenic lesions reflecting the fat tissue. Nuclear magnetic resonance of the left shoulder: adipose tissue accumulation, fatty focuses within the area of left deltoid attachments. Electromyography (deltoid): the record within the normal range. X-ray of both shoulders: slight densifications of bone structure around the neck of the left humerus and distal part of the right shoulder. Histopathological examination: diffused proliferation of normal fat tissue in subcutaneous tissue with an insignificant increase in the number of vessels. The image reflects lipomatosis. Consultation with a nephrology specialist: the patient with chronic renal failure, stage 3, constantly in the care of the Nephrology Department.

During hospitalization in the Clinic only an external treatment and UVA light therapy were applied on the common psoriasis focuses. The patient is in the care of the Dermatology, Nephrology and Hepatology Departments. The hospital set a date of a surgical consultation in order to establish a surgical fat tissue reduction schedule.

\section{Discussion}

Benign symmetric lipomatosis is a rare form of an excessive and irregular fat tissue layout. The aetiology remains unclear. However, it is believed that metabolic disorders related to alcohol abuse are as a crucial element of 
the above [3-5]. Nonetheless, as far as the described case is concerned, alcohol abuse was not reported.

Gu et al. [6] described a patient with diagnosed Madelung's disease, alcohol abuse reported in the medical file, an increased uric acid level, hyperinsulinemia and an increased level of hepatic enzymes. Our patient had increased levels of GGTP and amylase as well as disorders concerning renal functions in the course of chronic renal failure.

Kan et al. [7] presented 6 patients with benign symmetric lipomatosis. All of them had adipose tissue proliferation around the neck. In 1 patient, just like in the case of our patient, the deposits were also observed on the shoulders. All patients had left ventricular hypertrophy, which was also observed in the female patient we have just discussed. Two patients suffered from glucose intolerance, and one from diabetes, fatty liver and hyperuricemia. Three of them had episodes of sleep apnoea, typical of location of fat tissue excess around the neck. Five patients were addicted to alcohol. All patients underwent surgical removal of the fat tissue from the areas covered with excessive fat tissue and were advised to stop using alcohol. The disease recurred in 5 patients, during a period of 3 months to 4 years.

Goetze et al. [8] presented a 49-year-old female patient who was diagnosed with benign symmetric lipomatosis after a liver transplant due to decompensated cirrhosis. Fat tissue was mainly accumulated around the shoulders, neck and face. The patient gained $20 \mathrm{~kg}$.

Hirose et al. [9] also observed overweight and alcohol induced liver damage in a 46-year-old male patient. Zuber et al. [10] diagnosed benign symmetric lipomatosis in a 76-year-old patient who complained of muscle weakening in shoulders and lower limbs. Our patient did not report such symptoms. Fat tissue was arranged in a pseudo-athletic manner, on the shoulders, thighs and the torso. That female patient also suffered from arterial hypertension, diabetes, hyperlipidemia, hyperuricemia and polyneuropathy. The therapy involved pharmacotherapy based on Salbutamol, apart from surgical treatment.

As far as the case we have presented is concerned, fat tissue was accumulated mainly around shoulders. The literature presents several cases of other locations, which led to severe additional symptoms.

Ostrowski and Rubin [11] presented a patient with benign symmetric lipomatosis, which led to covering the larynx and to dysphonia. Milisavljevic et al. [12] described a case of a 58-year-old patient with Madelung's disease who suffered from respiratory disorders as a result of irregular fat tissue accumulation around the neck.

Ettl et al. [13] presented a 49-year-old patient with alcohol liver cirrhosis and an 11-year-long history of benign fatty lipomatosis. This patient suffered from symptoms of swallowing and speech disorders, next to hoarseness. It was clinically stated that the patient had asymmetric masses on both sides of the tongue. A histopathological examination confirmed the presence of satellite fat cells.
Similarly, Lopez-Ceres et al. [14] presented a 57-yearold female patient with benign symmetric lipomatosis. The patient had fat tissue on the neck, shoulders and thighs. The patient also suffered from impaired swallowing, speech and breathing. The tongue of the patient was considerably enlarged, which led to the above-mentioned disorders and to dental deformations. The patient had double-sided glossectomy. A histopathological examination revealed the presence of fat tissue without muscle tissue typical of the tongue.

A 60-year-old female patient described by Zubelewicz-Szkodzińska et al. [15] had fatty deposits around the neck, which considerably limited her mobility. Gdynia et al. [16] described a 55-year-old patient with Madelung's disease. He developed an axonal neuropathy and hyperlipoproteinemia. The authors suggest the occurrence of decreased COX activity in this patient and disorders within specific regions of mitochondrial tRNA.

Tekin and Ogetman [17] were the first ones to describe a central form of Madelung's disease, covering the lower part of the body, shoulders and the upper parts of thighs. We observed a quite similar, initial image in our patient. The treatment of benign symmetric lipomatosis consists mainly in fat tissue sucking or injection lipolysis. Hasegawa et al. [18] presented a case of a 42-year-old man with diagnosed benign symmetric lipomatosis. Mesotherapy treatment with phosphatidylcholine injections proved successful in this case. Pharmacological methods still remain controversial.

Zeitler et al. [19] described a 53-year-old male who gained $37 \mathrm{~kg}$ within 10 years. The patient had accumulated fatty masses surgically removed. The authors also administered fenofibrates in the dose of $200 \mathrm{mg} /$ day. Fibrates are antagonists of the alpha receptor activating peroxisome proliferation (PRAR- $\alpha$ ) and most probably evoke suppression of proteins responsible, among others, for fat tissue architecture [20].

In the case we have described above, the patient had a set of surgical consultations in order to establish a surgical fat tissue reduction schedule.

\section{Conclusions}

We have presented a case of a female patient with diagnosed Madelung's disease, without alcohol abuse. Such abuse is reported in $90 \%$ of patients with this disease. Nevertheless, the patient has other typical (and described in the literature) hepatic and pancreatic function impairments, but also glomerular nephritis, which very rarely co-exists with benign symmetric lipomatosis.

\section{Conflict of interest}

All authors declare no conflict of interest. 


\section{References}

1. Braun-Falco O, Plewig G, Wolff HH, et al. Fat tissue disease. In: Dermatology. Braun-Falco O, Plewig G, Wolff HH, et al. Czelej, Lublin 2010; 1146-7.

2. Rorke S, Lee M, Niumsawatt V, et al. Benign symmetric lipomatosis. Pathology 2009; 41: 600-2.

3. Kawakami T, Takeuchi S, Soma Y. Benign symmetric lipomatosis associated with atopic dermatitis and chronic alcohol abuse in a Japanese man. Acta Derm Venereol 2009; 89: 440-1.

4. Goshtasby P, Brooks G, Fielding LP. Lipomatous disorder of the peri-trochanteric soft tissue: case report and review. Curr Surg 2006; 63: 338-44.

5. Guilemany JM, Romero E, Blanch JL. An aesthetic deformity: Madelung's disease. Acta Otolaryngol 2005; 125: 328-30.

6. Gu W, Dou J, Yang G, et al. The endocrine and metabolic evaluation of benign symmetrical lipomatosis: a case report and literature review. Neuro Endocrinol Lett 2010; 31: 44650.

7. Kan $\mathrm{Y}$, Yao P, Xin W, et al. Recent progress on diagnosis and treatment of benign symmetric lipomatosis. Lin Chung Er Bi Yan Hou Tou Jing Wai Ke Za Zhi 2010; 24: 105-7.

8. Goetze S, Hommann M, Elsner P, Kaatz M. Induction of benign symmetric lipomatosis (Launois-Bensaude syndrome) in a female patient after liver transplantation. Dermatology 2008; 216: 337-40.

9. Hirose A, Okada Y, Morita E, Tanaka Y. Benign symmetric lipomatosis associated with alcoholism. Intern Med 2006; 45: 1001-5.

10. Zuber M, Pittasch D. Benign symmetric lipomatosis (Launois-Bensaude syndrome). A rare cause of muscular weakness. Eur J Med Res 2006; 11: 174-7.

11. Ostrowski KR, Rubin AD. Benign symmetric lipomatosis involving the supraglottic larynx: a rare cause of dysphonia. Otolaryngol Head Neck Surg 2011; 145: 360-1.

12. Milisavljevic D, Zivic M, Radovanović Z, Stankovic P. Severe dyspnea as atypical presenting symptom of Madelung's disease. Hippokratia 2010; 14: 133-5.

13. Ettl T, Gaumann A, Ehrenberg R, et al. Encapsulated lipomas of the tongue in benign symmetric lipomatosis. J Dtsch Dermatol Ges 2009; 7: 441-4.

14. Lopez-Ceres A, Aguilar-Lizarralde Y, Villalobos Sánchez A, et al. Benign symmetric lipomatosis of the tongue in Madelung's disease. J Craniomaxillofac Surg 2006; 34: 489-93.

15. Zubelewicz-Szkodzińska B, Danikiewicz A, Pietka-Rzycka A, et al. Madelung disease: case report. Pol Arch Med Wewn 2006; 115: 144-7.

16. Gdynia HJ, Sperfeld AD, Knirsch U, et al. Benign symmetric lipomatosis with axonal neuropathy and abnormalities in specific mitochondrial tRNA regions. Eur J Med Res 2006; 11: 545-6.

17. Tekin A, Ogetman Z. Central form of multiple symmetric lipomatosis: a case report. Cases J 2009; 2: 8427.

18. Hasegawa T, Matsukura T, Ikeda S. Mesotherapy for benign symmetric lipomatosis. Aesthetic Plast Surg 2010; 34: 153-6.

19. Zeitler H, Ulrich-Merzenich G, Richter DF, et al. Multiple benign symmetric lipomatosis: a differential diagnosis of obesity. Is there a rationale for fibrate treatment? Obes Surg 2008; 18: 1354-6.

20. Gutherc R, Jodkowska A, Drożdż K, et al. Case reports in daily medical practice - painful lipomatosis in Dercum disease. Postep Derm Alergol 2010; 27: 131-4. 